\title{
Difficulties with interpreting cytokine/chemokine values in disease states
}

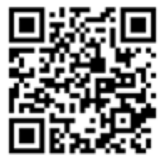

This edition of the journal features an interesting article on cytokine/chemokine profiles in HIV-exposed infants with hypoxic pneumonia. Whilst there are several interesting aspects, this paper highlights several aspects of biomarker measurements relevant to translational research. Thus, a closer look at the biomarker measurements is warranted.

Cytokines, chemokines and adhesion molecules are host-specific biomarkers that are present in health in humans. They are present at baseline concentrations (mean and range) and have natural variability. In disease states, there is either a premorbid change in biomarker levels that predisposes a person to acquisition of an illness or a responsive variation in concentrations with development of the illness. Biomarkers can therefore be utilised as diagnostic tools, and/or surrogate markers for monitoring the natural progression or response to an intervention. The measurement of biomarkers at different time intervals is likely to yield a pattern of response to the natural disease or to the intervention. Consequently, the measurement of cytokine and chemokine values in a cross-sectional manner without defining exactly the reason for the time point makes interpretation difficult.

Another concern with the measurement of biomarkers relates to the robustness of the technical analysis. While the Luminex multiplex technology by Bio-Rad Laboratory has been validated and accredited, there is a need to perform quality assurance of the results. Samples should be processed in duplicate and verified against those with predefined concentrations of cytokines/chemokines. This exercise is expensive.

A further consideration is the quality of the sample obtained and the preservation processes for storage of samples prior to testing. Biomarker levels in different bodily fluids are likely to be dependent on the site, nature and severity of the disease/illness at that location. In addition, biomarker values in different fluids may be impacted by the quality of the sample obtained. Sufficient good-quality sample is necessary to ensure accurate readings. Measurement of whole blood and plasma protein biomarkers (proteomics) can vary with the degree of anaemia or viscosity of the sample. Sputum biomarkers can vary due to the quality of the sample obtained. Breath volatile organic compounds (metabolites) measured by gas chromatography and mass spectrometry can vary with changes in flow volumes, while urine biomarkers could be affected by its composition and the presence of inhibitors. In order to adjust for these variables, a clear understanding about the degradability of biomarkers is necessary.

Disease states often induce multiple rather than single changes in biomarkers. Co-infections or co-diseases are likely to have an agonistic or antagonistic impact on levels of biomarkers measured. Acute-on-chronic diseases, such as exacerbations in bronchiectasis, could alter values of cytokines/chemokines obtained as compared with stable chronic disease. Subclinical infection will impede the correct interpretation of values obtained. The cost of measuring an individual specific biomarker is high, so most laboratory platforms utilise multiplex cytokine technology. Obtaining results about several cytokines/chemokines often confuses interpretation as the theoretical explanations are not congruent. The delay in obtaining the result often mitigates against its value as the patient's condition could have changed from the time of sampling. Point-of-care measurement of cytokines/chemokines would be useful in defining a response to the result but much more unequivocal data to guide clinical practice are required. Finally, cytokine responses of the host are modified by the levels of the stimulus provided by the pathogen, which is dependent on their load, the rate of reproduction and the rate of transcription.

\section{Prakash M Jeena}

Department of Paediatrics and Child Health, University of KwaZuluNatal, Durban, South Africa

S Afr Respir J 2016;22(1):2. DOI: 10.7196/SARJ.2016.v22i1.65 